\title{
Avaliação da compreensão de imagens de cartilha para pacientes sobre Artroplastia Total de Quadril
}

\author{
Evaluation of images of a booklet for patients about total hip arthroplasty
}

\author{
Katarina Miky Tsuzuki, Marcelo Cavalheiro de Queiroz, Nayra Deise dos Anjos Rabelo, \\ Walter Ricioli Jr, Giancarlo Polesello, Sara Miriam Goldchmit
}

Materiais educativos, Saúde, Artrose do Quadril, Imagens

Os materiais educativos na área da saúde contribuem para a recuperação dos pacientes submetidos a cirurgias de grande porte, como por exemplo, a Artroplastia Total de Quadril (ATQ), realizada em casos graves de artrose. Entretanto, a inadequação da apresentação do conteúdo diminui a eficácia da comunicação. Uma possível estratégia de comunicação para melhorar a adesão e compreensão desses materiais é o uso de imagens, essenciais para a visualização de informações abstratas de difícil entendimento pelo público leigo. O presente artigo busca explorar a percepção e compreensão das imagens presentes na cartilha fornecida pela Santa Casa de Misericórdia de São Paulo aos pacientes antes da realização da ATQ. Utilizando métodos qualitativos, foram coletados dados de 9 pacientes atendidos pela Santa Casa, através de entrevista semiestruturada sobre compreensão das imagens. Apesar de as imagens da cartilha agradarem a maioria dos participantes, os resultados indicam dificuldades na compreensão de alguns elementos das figuras e incoerências entre a interpretação dos entrevistados e a instrução proposta pelo material.

Health Education Material, Health, Hip Osteoarthritis, Images

Health education materials contribute to patient's recovery from major surgeries, such as the Total Hip Arthroplasty (THA), done in severe cases of arthritis. However, an inappropriate presentation of the content may decrease its efficacy. A possible communication strategy to improve adherence and comprehension of these materials is to use images, which are essential to simplify the visualization of abstract and complex information by the public. This paper aims to explore the perception and comprehension of the images in the booklet provided by Santa Casa de Misericórdia de São Paulo before the THA by the patients of the institution. Using qualitative methods, data of 9 patients treated in Santa Casa was collected by using semi-structured interviews about the comprehension of the images. Even though the pictures pleased most of the participants, the findings indicate discrepancies between the interpretation of some pictures by the interviewees and the original purpose of the booklet's instructions.

\section{Introdução}

A atuação do Design da Informação (DI) na área da saúde pode contribuir para a promoção do bem-estar de diversas maneiras, como por exemplo, através dos materiais educativos, já que um dos grandes desafios enfrentados pelos profissionais da saúde é garantir a clareza na

Anais do $10^{\circ} \mathrm{CIDI}$ e $10^{\circ} \mathrm{CONGIC}$

Kelli C.A.S. Smythe, Rafael de Castro Andrade (orgs.)

Sociedade Brasileira de Design da Informação - SBDI

Curitiba | Brasil | 2021
Proceedings of the $10^{\text {th }} \mathrm{CIDI}$ and $10^{\text {th }}$ CONGIC

Kelli C.A.S. Smythe, Rafael de Castro Andrade (orgs.)

Sociedade Brasileira de Design da Informação - SBDI Curitiba | Brazil | 2021 
comunicação com os pacientes (Noël et al., 2016). Estudos apontam que os conhecimentos sobre Design da Informação podem facilitar a interação entre os usuários e informações complexas (Black et al., 2017). No entanto, muitos artefatos voltados para comunicação em saúde não empregam boas práticas de DI para a apresentação do conteúdo, o que dificulta a compreensão pelo leitor (Abraham et al., 2012).

A equipe do Departamento de Ortopedia e Traumatologia da Santa Casa de Misericórdia de São Paulo, parceira do presente projeto de pesquisa, oferece um manual de orientações e cuidados (figura 1 e figura 2) para auxiliar os pacientes que serão submetidos à Artroplastia Total de Quadril (ATQ), uma cirurgia para a implantação de uma prótese que pode ser necessária em casos graves de artrose (Hunter \& Bierma-Zeinstra, 2019). Tal iniciativa é válida ao considerar benefícios proporcionados por essa intervenção, como a redução da ansiedade, manejo de expectativas e dor em processos cirúrgicos (Paiva et al., 2017).

Figura 1: Capa da cartilha enviada aos pacientes (@ Darlon Studio)

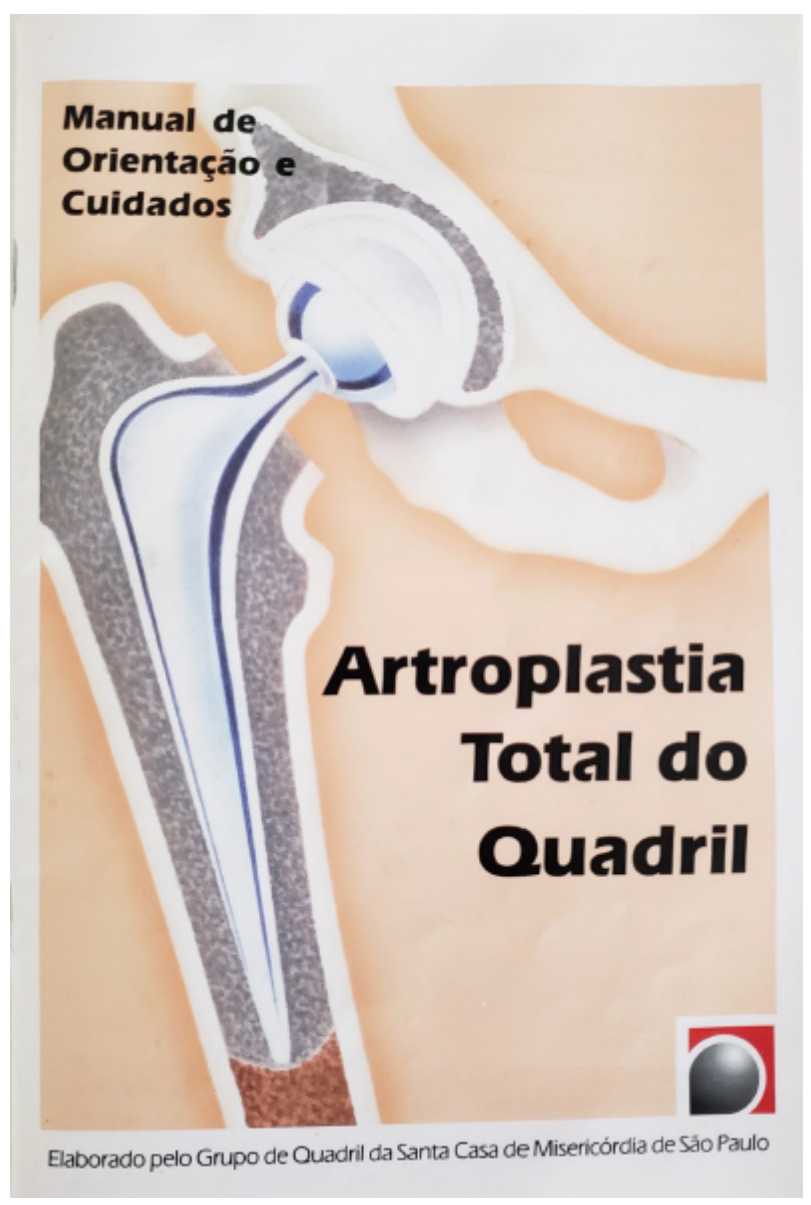


Figura 2: Página dupla da cartilha (@ Darlon Studio)

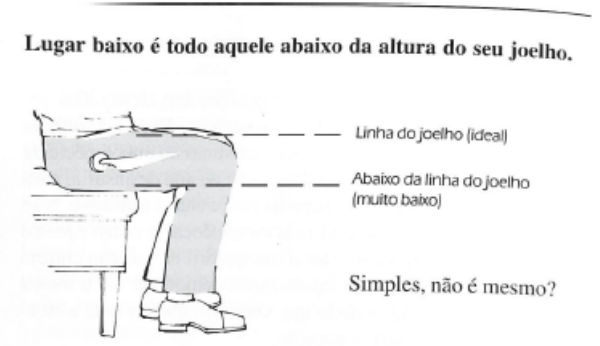

Sāo nestes locais, considerados BAIXOS, que acontecem as luxaçōes das próteses, e os três locais mais comuns são:

\section{1- VASO SANITÁRIO:}

O pior local, pois você quase sempre estará sozinho

Para evitar esta complicação, existem os dispositivos que elevam a altura do assento e que são capazes de evitar que você venha com o tronco para a frente ao levantar-se, pois são mais altos que seus joelhos. Não hesite em pedir ajuda a algum familiar para levantar-se até que aprenda a melhor forma fazê-lo sozinho.

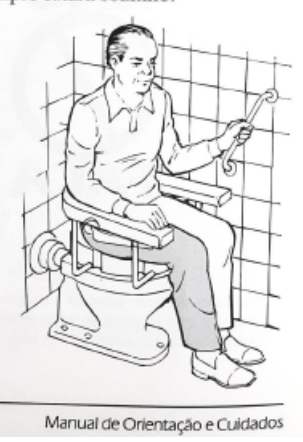

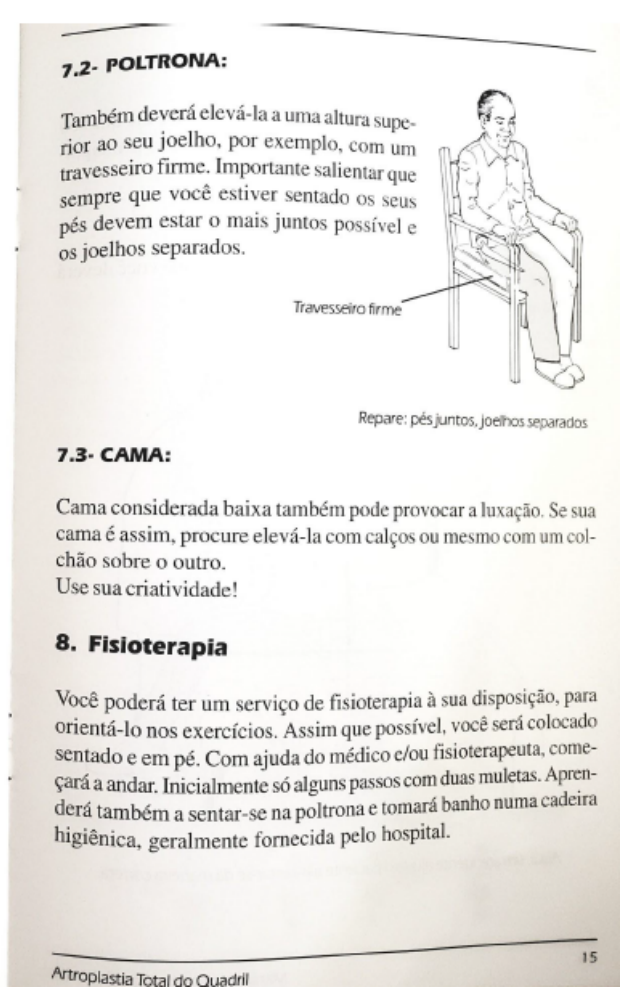

Apresentar as informações utilizando figuras pode ajudar os usuários a compreenderem melhor as informações e a relembrarem os assuntos apresentados no material. Todavia, os pacientes podem ignorar partes do texto e tentar adivinhar o significado das imagens, criando espaço para interpretações equivocadas e distintas das intenções dos autores (Houts et al., 2006).

Em vista da emergente necessidade de aprimorar a comunicação entre profissionais da saúde e o público leigo, o presente artigo tem como objetivo analisar a compreensão das imagens da cartilha "Manual de Orientações e Cuidados: Artroplastia total de quadril" pelos pacientes do Ambulatório de Quadril da Santa Casa de São Paulo.

\section{Método}

Inicialmente, foi conduzida uma revisão bibliográfica exploratória sobre os temas saúde, Design da Informação e materiais educativos na área da saúde para gerar uma familiarização com o assunto. Em seguida, elaboramos o roteiro das entrevistas semiestruturadas adaptado de Cuperus et al (2013) e Abraham et al (2012). O roteiro foi revisado e validado pela equipe médica da Santa Casa.

Os pacientes foram selecionados segundo amostra de conveniência, conforme agenda de consultas ambulatoriais coincidentes com o período da pesquisa. O critério de inclusão foi estar na fila para a cirurgia de ATQ primária. Os pacientes selecionados foram contactados por telefone e convidados a participar do estudo. A cartilha foi então enviada por correio para estes 
pacientes que foram entrevistados por ligação telefônica em uma data de sua preferência após uma leitura prévia do material. Além disso, o aplicativo WhatsApp foi utilizado para mostrar as imagens durante a chamada. Na primeira etapa da entrevista, os participantes foram orientados a explicar como compreenderam as 5 imagens que representam contraindicações no processo de recuperação, sem poder consultar a cartilha naquele momento. Em uma segunda parte, foram feitas questões sobre a opinião geral dos participantes em relação às figuras.

Todas as entrevistas foram gravadas, transcritas e analisadas a partir do agrupamento de anotações feitas segundo áreas temáticas de interesse da pesquisa, tal como propõe Portigal (2013).

\section{Resultados}

As entrevistas foram realizadas entre novembro de 2020 e março de 2021. Ao total, 9 pacientes ( 8 mulheres e 1 homem) de idade média de 57,7 anos foram entrevistados.

No geral, a maioria dos entrevistados identificaram que as orientações se tratavam de contraindicações. Entretanto, um dos participantes não notou a observação "Não faça" no canto inferior esquerdo das figuras, o que o levou a errar a noção de proibição atribuída a todas as ilustrações. Quanto à figura 3, a maioria dos entrevistados compreendeu a orientação de "Não cruze as pernas"

Figura 3: Imagem da orientação "Não Cruze as pernas" (@ Darlon Studio)

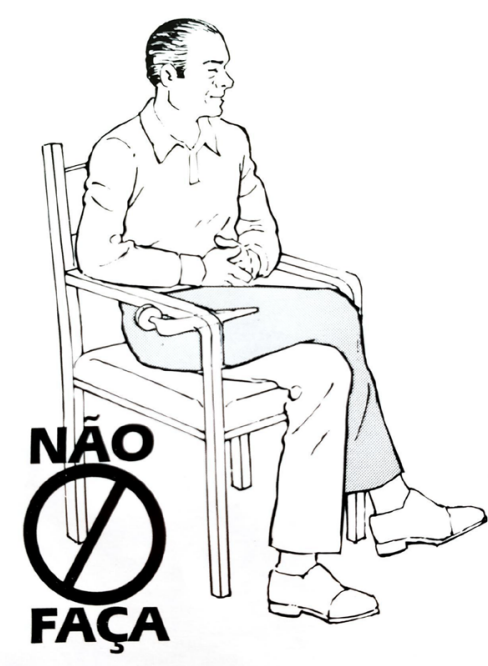

Alguns entrevistados confundiram o ato de agachar da figura 4 com levantar ou inclinar 0 corpo para frente. Além disso, foram mencionadas dúvidas em relação ao ângulo de $90^{\circ}$. 
Figura 4: Imagem da orientação "Não agache para apanhar nada no chão" (@ Darlon Studio)

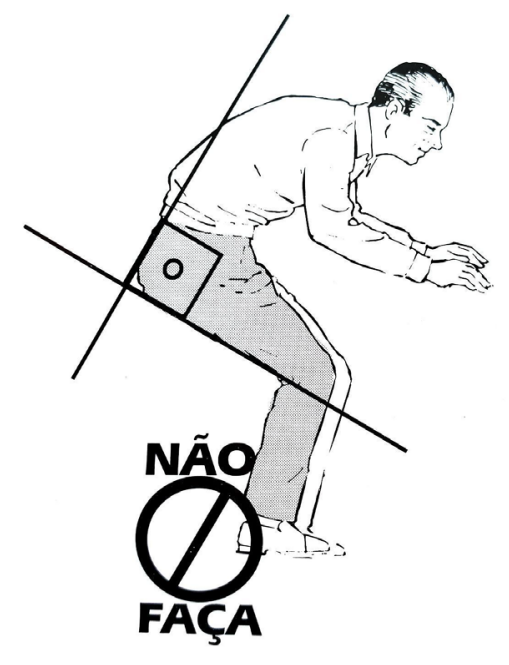

A instrução proposta pela figura 5 foi compreendida pelos entrevistados no geral, porém, também foram apresentadas dificuldades iniciais para identificar o movimento da seta.

Ademais, alguns entrevistados citaram as bengalas em suas respostas, um elemento que gerou confusões sobre a instrução original em alguns casos, pois a proibição do movimento foi associada à situação de usar bengalas.

Figura 5: Imagem da orientação "Não gire o membro para fora” (@ Darlon Studio)

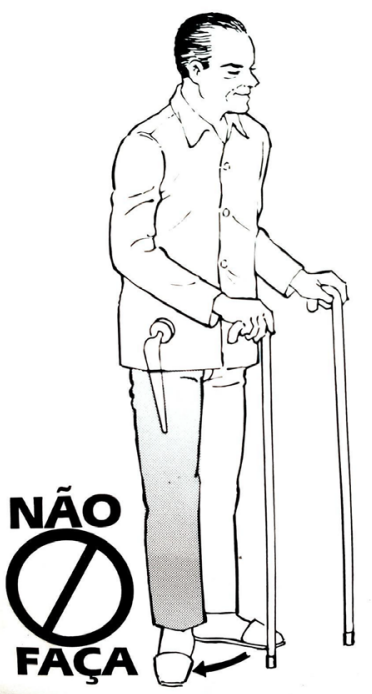

A essência da orientação da figura 6 foi compreendida pela maioria dos participantes. Todavia, o elemento extra representado pelo computador demandou um maior esforço do usuário para entender a figura e distraiu ou confundiu os entrevistados, uma vez que alguns relacionavam a proibição com a ação de "estar no computador". 
Figura 6: Imagem da orientação "Não dobre o quadril ou sente em lugar baixo" (@ Darlon Studio)

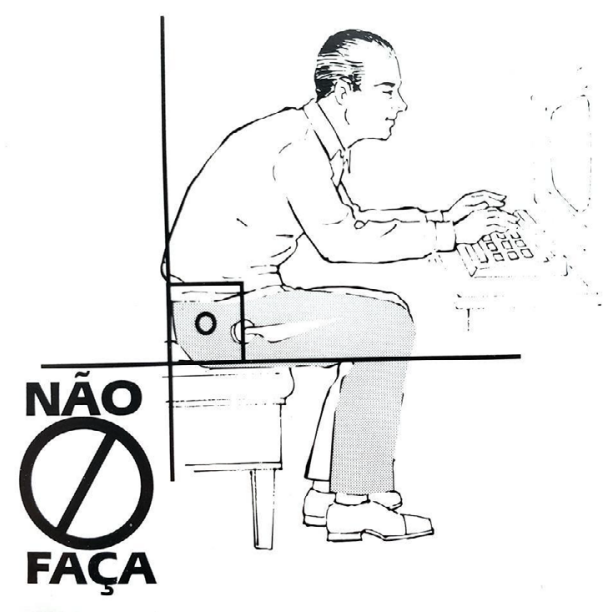

Metade dos entrevistados compreenderam a orientação da figura 7 "A posição que pode provocar a luxação de uma prótese é a flexão acima de $90^{\circ}$ e rotação interna. Cuidado maior nas poltronas sem braço" ou "Cuidado ao levantar-se!". Aqueles que erraram, confundiram a ação em questão com o ato de "se levantar rapidamente".

Figura 7: Imagem da orientação "Não dobre o quadril ou sente em lugar baixo" (@ Darlon Studio)

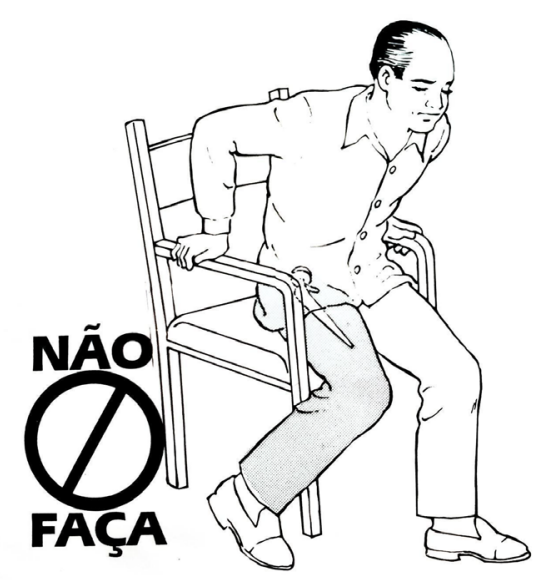

\section{Discussão}

Embora, em seus relatos, os entrevistados afirmam que as imagens estejam claras, os resultados apresentados indicam que a maioria dos participantes não compreenderam plenamente as informações apresentadas nas ilustrações. Kools (2012) afirma que tal situação não é incomum pois o entrevistado pode se sentir desconfortável em dizer que não entendeu algo ou ter uma falsa sensação de que compreendeu o assunto.

Apesar de Houts et al. (2006) e Wright (2012) indicarem que o uso de um desenho composto de linhas em preto e branco seja efetivo para materiais educativos na área da saúde, inserir alguns recursos ou omitir outros poderiam evitar algumas confusões apresentadas nas entrevistas. 
A inclusão do uso de cores, por exemplo, quando feita de maneira adequada, é capaz de chamar a atenção do leitor para aspectos específicos (Lupton \& Phillips, 2008; Wright, 2012), o que poderia facilitar a navegação na imagem pelo olhar do usuário. Outro ponto a ser considerado é o significado das cores (Heller, 2013), como o vermelho, que poderia reforçar a ideia de proibição.

Um recurso gráfico pouco usado na cartilha mas que poderia ser explorado para esclarecer certos movimentos são as setas (Souza \& Cunha, 2010), que poderiam clarificar as diferenças entre o movimento de "agachar" e o de "levantar" na figura 2. No entanto, também é imprescindível garantir um dimensionamento e posicionamento adequado dos símbolos pois, como foi apresentado nos resultados, tais sinais podem não ser perceptíveis se inseridos de maneira discreta.

Como explicado por Houts et al. (2006) e evidenciado pelos comentários dos entrevistados, aspectos menos relevantes poderiam ser suprimidos visto que estes podem distrair os leitores, interferir e até mesmo dificultar a compreensão das figuras. Tal situação, no caso do presente estudo, pode ser identificada pelo foco dos participantes em elementos desnecessários como o computador da figura 4 e as muletas da figura 3.

\section{Conclusão}

Os resultados deste estudo qualitativo de caráter multidisciplinar evidenciaram as dificuldades e benefícios do uso de imagens para a compreensão da cartilha sobre Artroplastia Total de Quadril oferecida pela Santa Casa de São Paulo pelos seus pacientes. A perspectiva dos usuários sobre o material existente pode contribuir para o desenvolvimento de uma nova cartilha que considere as necessidades informacionais e capacidades cognitivas do público, além de parâmetros técnicos do design da informação, promovendo maior eficácia na comunicação médico-paciente no contexto da saúde pública.

\section{Referências}

Abraham, C., Kools, M. (2012). Introduction: steps towards writing effective educational text. In Abraham, C., \& Kools, M. (Eds.). Writing Health Communication: An Evidence-Based guide (1st ed., pp 1-5). SAGE publications Itda.

Black, A., Luna, P., Lund, O., \& Walker, S. (2017). Information design: Research and practice (1st ed.). Routledge.

Cuperus, N., Smink, A. J., Bierma-Zeinstra, S. M., Dekker, J., Schers, H. J., De Boer, F., Van Den Ende, C. H., \& Vliet Vlieland, T. P. (2013). Patient reported barriers and facilitators to using a self-management booklet for hip and knee osteoarthritis in primary care: Results of a qualitative interview study. BMC Family Practice, 14, 1-10. https://doi.org/10.1186/14712296-14-181

Heller, E. (2013). A psicologia das cores: Como as cores afetam a emoção e a razão (1st ed.). Gustavo Gili. 
Houts, P. S., Doak, C. C., Doak, L. G., \& Loscalzo, M. J. (2006). The role of pictures in improving health communication: A review of research on attention, comprehension, recall, and adherence. Patient Education and Counseling, 61(2), 173-190. https://doi.org/10.1016/j.pec.2005.05.004

Hunter, D. J., \& Bierma-Zeinstra, S. (2019). Osteoarthritis. The Lancet, 393(10182), 1745-1759. https://doi.org/10.1016/S0140-6736(19)30417-9

Kools, M. (2012). Make written material easy to understand. In Abraham C., \& Kools, M. (Eds.). Writing Health Communication: An Evidence-Based guide (1st ed.; pp. 23-42). SAGE publications.

Lupton, E., \& Phillips, J. C. (2008). Novos fundamentos do Design. Cosac Naify.

Noël, G., Frascara, J., Campbell-Scherer, D., Chiapponi, M., Corcoran, H., Davidson Nursing, S., Dempster Fraser Health, L., Wes Ervin, C., Francescutti, L., Friedman, K., Gregory, J., Jones, P., Koskinen, I., Lawrence Alberta Health Services, J., Robert Lederer, C., Norman, D., Pitt, M., Predan, B., Sadler Takach, B., Zender Design, M. (2016). Health and Design Fostering a culture of collaboration through education. August.

Paiva, B. C., Sousa, C. S., Poveda, V. B., \& Turrini, R. N. T. (2017). Avaliação da efetividade da intervenção com material educativo em pacientes cirúrgicos: revisão integrativa da literatura. Revista SOBECC, 22(4), 208. https://doi.org/10.5327/z1414-4425201700040006

Portigal, S. (2013). Analyzing and Synthesizing Your Interview Data. In Interviewing User, How to uncover compelling insights (pp. 136-141). Rosenfeld media.

Souza, J., \& Cunha, R. (2010). O design de gráficos auxiliares na representação de movimento para fins instrucionais. Anais do $9^{\circ}$ Congresso Brasileiro de Pesquisa e Desenvolvimento Em Design.

Wright, P. (2012). Using graphics effectively in text. In Abraham C., Kools, M. (Eds.). Writing Health Communication: An Evidence-Based guide (ed.; pp. 62-82). SAGE publications Itda.

\section{Sobre os autores}

Katarina Miky Tsuzuki, graduanda, USP, Brasil <kttsuzuki@usp.br>

Marcelo Cavalheiro de Queiroz, mestre, Santa Casa de São Paulo, Brasil

<macqueiroz@yahoo.com>

Nayra Deise dos Anjos Rabelo, doutora, Universidade Nove de Julho - UNINOVE, Brasil

<nayrarabelohip@gmail.com>

Walter Ricioli Junior, doutor, Santa Casa de São Paulo, Brasil <walterricijr@yahoo.com.br>

Giancarlo Polesello, doutor, Santa Casa de São Paulo, Brasil

<giancarlopolesello@hotmail.com>

Sara Miriam Goldchmit, doutora, USP, Brasil <saragold@usp.br> 